\title{
17ß-Estradiol and Tamoxifen Prevent the Over-Glycosylation of Rat Trabecular Bone Collagen Induced by Ovariectomy ${ }^{1}$ )
}

\author{
Luigi Moro, Paolo Bettica, Milena Romanello and Karem Noris Suarez
}

Dipartimento di Biochimica, Biofisica e Chimica delle Macromolecole, Università degli Studi di Trieste, Trieste, Italy

Summary: We have recently demonstrated that ovariectomy in the rat causes over-glycosylation of collagen which is restricted to trabecular bone. In order to obtain further evidence, we studied whether estrogen or tamoxifen treatment prevented over-glycosylation of trabecular bone collagen. Forty one-hundred-day-old female rats were subjected to ovariectomy $(n=30)$ or sham-operation $(n=10)$. Starting the day of the operation, sham-operated rats were treated with vehicle, while ovariectomized rats were divided into three groups and treated with vehicle $(n=10)$, estrogen $(n=10)$ or tamoxifen $(n=10)$. Five rats from each group were sacrificed at 115 and 145 days of age. Femurs and tibiae were separated into cortical and trabecular bone, demineralized, hydrolyzed and analyzed by HPLC for hydroxylysine glycosides and hydroxyproline content. Hydroxylysine glycoside content was expressed as a molar ratio with hydroxyproline. The results can be summarized as follows:

1) cortical bone collagen glycosylation did not vary among the different groups;

2) over-glycosylation of trabecular bone collagen observed in the ovariectomized rats was prevented by the administration of either $17 \beta$-estradiol or tamoxifen.

These data demonstrated that estrogens affect glycosylation of trabecular bone collagen.

\section{Introduction}

Collagen is the main structural protein of the extracellular matrix, which plays a crucial role in maintaining the skeleton's biological function. The intracellular posttranslational modifications involved in biosynthesis of type I collagen include hydroxylation of prolyl and lysyl residues to hydroxyproline and hydroxylysine, and glycosylation of some of the hydroxylysine residues with galactose or glucosylgalactose.

In a recent work (1), we have shown that ovariectomy causes an increase in bone collagen glycosylation, suggesting that estrogens may influence bone matrix. Interestingly, the increase in collagen glycosylation was seen only in trabecular bone. These findings would seem to be relevant because an increase in glycosylated hydroxylysine causes a steric hindrance that decreases fibril diameter, such as is observed in osteogenesis imperfecta, a heritable disease characterized by bone brittleness (2). Thus a change in collagen could lead to a change in bone, and in turn, the traditionally accepted definition of osteoporosis as "too little bone" might seem inadequate because osteoporosis may not be just a

\footnotetext{
1) Funding organizations: Italian Ministry of University and of Scientific and Technological Research (MURST) and Research National Council (CNR).
}

matter of too little bone, but also that the bone has different chemical-physical properties which may influence mechanical properties.

Although ovariectomy mainly leads to an estrogen depletion, it also induces a decrease in progesterone and testosterone serum concentration, both of which are known to act on bone metabolism $(3,4)$. In order to provide direct evidence on the relationship between collagen glycosylation of trabecular bone and estrogens, the ovariectomized animals were treated with either $17 \beta$ estradiol or with the partial agonist of the estrogen receptor, tamoxifen $(5,6)$. This paper, which is a continuation of the previous one (1), reports the results obtained in this investigation.

\section{Materials and Methods}

Animals and treatment

All groups of animals were kept in identical conditions and were allowed free access to food and water. The surgical procedures were carried out in accordance with institutionally approved small animals protocols.

Forty female Sprague-Dawley rats, one hundred days old, were subjected to either ovariectomy $(n=30)$ or sham operation $(\mathrm{n}=10)$. Bilateral ovariectomies were carried out under anaesthetic (xylazine hydrochloride, $0.15 \mathrm{ml} / \mathrm{kg}$ of body weight, plus ketamine hydrochloride, $1 \mathrm{ml} / \mathrm{kg}$ of body weight) using a dorsal approach. After surgery ovariectomized animals were divided at random into 3 groups of 10 animals: the first group was treated 
with vehicle only, the second with estrogen and the third with tamoxifen. The sham-operated animals were treated with vehicle only. The vehicle consisted of a $70 \%$ ethanol solution diluted $1: 20$ with sesame oil. Treatment was carried out by subcutaneous injection once a week starting the day of the operation. All treatments were freshly prepared immediately before injection. 17ß-Estradiol (Sigma, Italy) was first dissolved in $70 \%$ ethanol $(6.0 \mathrm{~g} / 1)$ and then diluted $1: 20$ with sesame oil in order to obtain a final concentration of $0.3 \mathrm{~g} / \mathrm{l}$. Tamoxifen (tamoxifen citrate, Sigma, Italy) was dissolved in $70 \%$ ethanol in a concentration of $20 \mathrm{~g} / \mathrm{l}$. The dose of estrogen was $300 \mu \mathrm{g} / \mathrm{kg}$ of body weight, while that of tamoxifen was $20 \mathrm{mg} / \mathrm{kg}$ of body weight. Injection volume was $1 \mathrm{ml} / \mathrm{kg}$ of body weight.

\section{Tissue preparation}

Five rats from each group were sacrificed under anaesthesia at 115 and 145 days of age and tibiae and femurs were removed and carefully dissected free of adhering soft tissue. After washing with water, bones were dissected into three anatomically distinct areas: the diaphysis containing only cortical bone and the proximal and distal metaphysis, from which trabecular bone was scraped and pooled. The periosteum was stripped from the diaphysis and cortical bone was fragmented.

\section{Bone treatment prior to analyses}

Samples were defatted by sequential treatment in methanol for $16 \mathrm{~h}$ followed by chloroform/methanol ( $2: 1$ by vol.) for $48 \mathrm{~h}$ and methanol for a further $16 \mathrm{~h}$ and decalcified at $4{ }^{\circ} \mathrm{C}$ as previously described (7). Subsequently, bones were extracted with $4 \mathrm{~mol} / \mathrm{l}$ guanidine hydrochloride, to remove soluble matrix macromolecules from the largely insoluble collagenous matrix. The material thus obtained was freeze-dried and then hydrolyzed in $2.5 \mathrm{~mol} / \mathrm{l} \mathrm{NaOH}$ (bone dry weight, $15 \mathrm{~g} / \mathrm{l}$ ) for $22 \mathrm{~h}$ and hydroxyproline and hydroxylysine glycosides were measured.

\section{Hydroxyproline measurements}

Hydroxyproline measurements were performed by HPLC following the method of Teerlink et al. (8). Hydroxyproline concentration was determined from the ratio between its peak height and the peak height of the internal standard 3,4-dehydro- $L$-proline (Fluka BioChemika, Switzerland). Hydroxyproline content is expressed as mmol of hydroxyproline per $\mathrm{g}$ of bone dry weight.

\section{Hydroxylysine glycoside measurements}

Hydroxylysine glycosides were measured as previously reported (9). Briefly: aliquots of hydrolyzed tissue were derivatized using dansyl chloride following the method of Gray (10), and $20 \mu \mathrm{l}$ were injected into a reversed-phase Ultrasphere-ODS $\left(\mathrm{C}_{18}\right.$ of $5 \mu \mathrm{m}$ particles) HPLC column, of $250 \times 4.6 \mathrm{~mm}$ i.d. The HPLC Beckman Gold Model was connected with a fluorimeter (Shimadzu RF551), using an excitation wavelength of $366 \mathrm{~nm}$ and an emission wavelength of $490 \mathrm{~nm}$. The area of the peaks was calculated by an IBM personal computer. Values of hydroxylysine glycosides were calculated using $L$-lysine as an external standard as previously described (11). Hydroxylysine glycoside content is expressed as $\mathrm{mmol} / \mathrm{mol}$ of hydroxyproline.

\section{Statistical analysis}

Statistical differences between groups were evaluated both by one factor and two factor analysis of variance (ANOVA). In the two factor ANOVA the two factors analyzed were age and treatment. The significance of the difference of the mean between groups was estimated by Scheffè test.

\section{Results}

\section{Weight gain}

The body weight of the four groups of rats was similar at the beginning of the experiment. As shown in figure

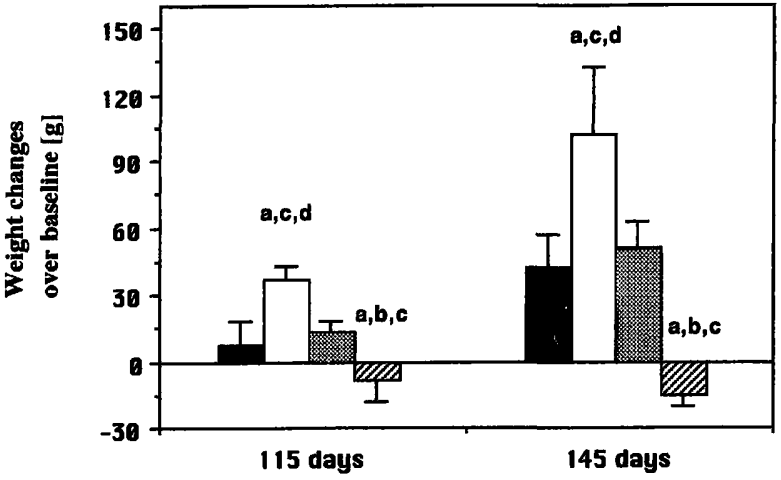

Fig. 1 Rat's body weight. Variations from baseline values in body weight for the four treatment groups ( $\square$ sham-operated, $\square$ ovariectomized, $\square$ ovariectomized treated with $17 \beta$-estradiol and $\square$ ovariectomized treated with tamoxifen) at 115 and 145 days of age $(\mathrm{a}=$ $\mathrm{p}<0.05$ vs. sham-operated; $\mathrm{b}=\mathrm{p}<0.05$ vs. ovariectomized; $\mathrm{c}=$ $\mathrm{p}<0.05$ vs. ovariectomized treated with $17 \beta$-estradiol; $\mathrm{d}=$ $\mathrm{p}<0.05$ vs. ovariectomized treated with tamoxifen).

1, the sham-operated rats showed a gain in body weight both at 115 days $(+8.2 \pm 10.3 \mathrm{~g})$ and at 145 days $(+42.8 \pm 14.1 \mathrm{~g})$ of age. A significantly larger increase in body weight was seen in the ovariectomized rats. The ovariectomized rats gained $37.2 \pm 5.8 \mathrm{~g}$ at 115 days of age $(\mathrm{p}<0.05$ vs. sham-operated) and $102.8 \pm 30.2 \mathrm{~g}$ at 145 days of age $(p<0.05$ vs. sham-operated). Treatment with $17 \beta$-estradiol completely prevented the increase in body weight gain caused by ovariectomy (115 days: + $13.6 \pm 5.5 \mathrm{~g}$, NS vs. sham-operated, $\mathrm{p}<0.05$ vs. ovariectomized; 145 days: $+51 \pm 11.7 \mathrm{~g}$, NS vs. sham-operated, $p<0.05$ vs. ovariectomized). Treatment with tamoxifen caused a decrease in body weight (115 days: $-8.2 \pm 9.5, \mathrm{p}<0.05$ vs. sham-operated, ovariectomized and ovariectomized treated with 17ß-estradiol; 145 days: $-14.4 \pm 5.0, \mathrm{p}<0.05$ vs. sham-operated, ovariectomized and ovariectomized treated with $17 \beta$-estradiol).

\section{Proline hydroxylation}

The hydroxyproline content of cortical and trabecular bone collagen was not significantly different (data not shown), as previously reported (12).

\section{Collagen glycosylation of cortical bone}

The hydroxylysine glycoside content of cortical bone collagen did not change at the two different time points in the sham-operated rats. Likewise, the hydroxylysine glycoside content of cortical bone collagen was similar in all four groups of rats at both times of the experiment (tab. 1 and fig. 2).

\section{Collagen glycosylation of trabecular bone}

The hydroxylysine glycoside content of trabecular bone collagen did not change at the two different time points in the sham-operated rats. On the contrary, ovariectomy 
Tab. 1 Two factors ANOVA of the changes in cortical bone collagen hydroxylysine glycoside content in sham-operated, ovariectomized, ovariectomized treated with $17 \beta$-estradiol and ovariecto- mized treated with tamoxifen rats at 115 and 145 days of age. The two factors analyzed were treatment (A) and age (B).

\begin{tabular}{lccccc}
\hline Source & Degrees of freedom & Sum of squares & Mean square & F-test & P value \\
\hline Treatment (A) & 3 & 44.588 & 14.863 & 1.056 & 0.3868 \\
Age (B) & 1 & 39.434 & 39.434 & 2.802 & 0.1077 \\
AB & 3 & 13.167 & 4.389 & 0.312 & 0.8166 \\
Error & 32 & 323.639 & 14.071 & & \\
\hline
\end{tabular}

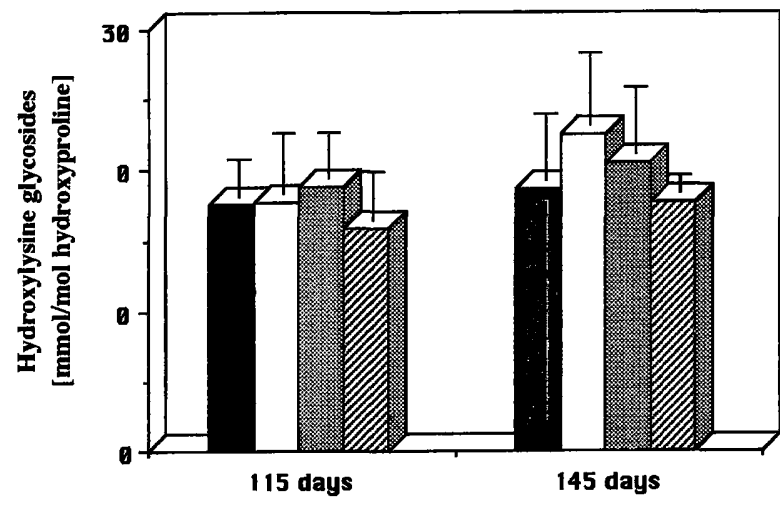

Fig. 2 Cortical bone collagen glycosylation. Hydroxylysine glycoside content in the four treatment groups ( $\square$ sham-operated, $\square$ ovariectomized, $\square$ ovariectomized treated with $17 \beta$-estradiol and $\mathbb{Z}$ ovariectomized treated with tamoxifen) at 115 and 145 days of age.

caused a significant increase in hydroxylysine glycoside content of trabecular bone collagen $(p<0.05)$. Treatment with both estrogen and tamoxifen prevented the over-glycosylation of trabecular bone collagen caused by castration. In ovariectomized rats treated with $17 \beta-$ estradiol, the hydroxylysine glycoside content of trabecular bone was similar to that of sham-operated rats and was significantly lower than that of ovariectomized rats $(\mathrm{P}<0.05)$. Likewise, in ovariectomized rats treated with tamoxifen the hydroxylysine glycoside content of trabecular bone was similar to that of sham-operated rats and was significantly lower than that of ovariectomized rats $(p<0.05)$. There was no significant difference in trabecular bone collagen glycosylation between ovariectomized rats treated with either $17 \beta$-estradiol or tamoxifen (tab. 2 and fig. 3).

\section{Discussion}

It is known that ovariectomy in the rat causes an increase in weight gain and that this is completely pre-

Tab. 2 Two factors ANOVA of the changes in trabecular bone collagen hydroxylysine glycoside content in sham-operated, ovariectomized, ovariectomized treated with $17 \beta$-estradiol and ovariec- vented by estrogens and tamoxifen (5). This is confirmed in our study where body weight gain was significantly higher in ovariectomized rats as compared to sham-operated controls. Furthermore, 17 $\beta$-estradiol treatment completely prevented an increased growth rate, while tamoxifen caused a significant weight loss. Moreover, although the uterine weight was not measured at the autopsy, the organ was clearly atrophied in all the ovariectomized rats, while as expected, in sham-operated rats the uterus was normal. Therefore, these data strongly support a successful ovariectomy and a correct dosage of estrogens and tamoxifen even if estrogen serum concentration was not measured.

The data derived from this experiment clearly show that both estrogen and tamoxifen are capable of preventing the effects of ovariectomy on the degree of collagen glycosylation of trabecular bone within 15 days from the removal of the ovaries. At 45 days from surgery the

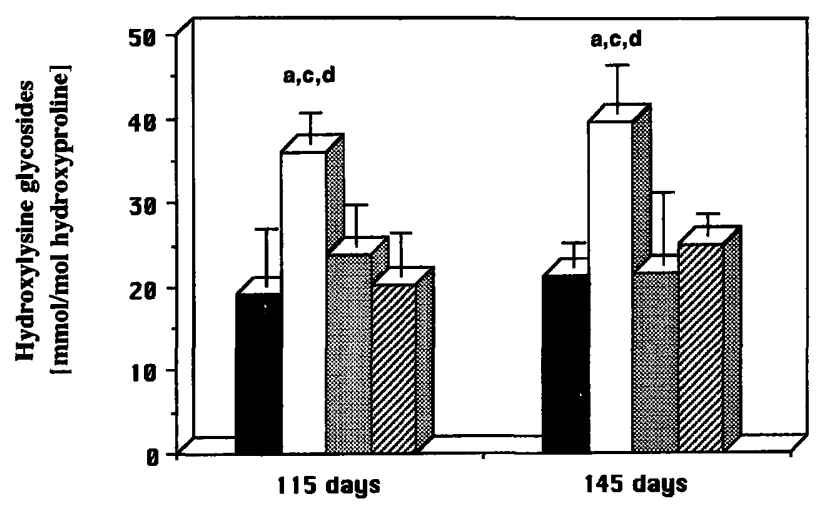

Fig. 3 Trabecular bone collagen glycosylation. Hydroxylysine glycoside content in the four treatment groups (- sham-operated, $\square$ overiectomized, $\square$ ovariectomized treated with $17 \beta$-estradiol and ovariectomized treated with tamoxifen) at 115 and 145 days of age $(a=p<0.05$ vs. sham-operated; $c=p<0.05$ vs. ovariectomized treated with $17 \beta$-estradiol; $d=p<0.05$ vs. ovariectomized treated with tamoxifen).

tomized treated with tamoxifen rats at 115 and 145 days of age. The two factors analyzed were treatment (A) and age (B).

\begin{tabular}{lccccc}
\hline Source & Degrees of freedom & Sum of squares & Mean square & F-test & P value \\
\hline Treatment (A) & 3 & 1449.943 & 483.314 & 17.491 & 0.0001 \\
Age (B) & 1 & 30.163 & 30.163 & 1.092 & 0.307 \\
AB & 3 & 55.99 & 18.663 & 0.675 & 0.5759 \\
Error & 32 & 635.557 & 27.633 & & \\
\hline
\end{tabular}


same effect is still observed for both drugs (fig. 3). On the contrary, cortical bone does not seem to be influenced either by ovariectomy or by replacement therapy (fig. 2). This consideration would suggest that synthesis and secretion of the collagenous matrix by cortical osteoblasts is either not influenced or influenced in a different way by these systemic factors. Whether this is due to a suppressed or a defective expression of the estrogen receptor, or because the collagen turnover rate of cortical bone is lower than that of trabecular bone collagen, cannot be established at the moment. However, it should be considered that the microenvironment is different in the two bone compartments and that this plays an important role in the recruitment and differentiation of osteoblast precursor cells as well as in the activation of mature cells (13).

An important point raised by this finding is that ovariectomy induces a modification of the trabecular bone matrix which is prevented by replacement treatment. To this point, as also suggested by the literature (14-17), it is worth noting that a changed matrix is certainly relevant to the biological features of bone. Although the main difference between trabecular and cortical bone is the much greater surface area of the former, which explains the differences in bone turnover rates between cortical and trabecular bone, the hypothesis that a modified and possibly defective matrix could somehow be recognized and further removed by osteoclasts cannot be excluded. Thus there might be a mechanism of osteoclast activation and matrix recognition, perhaps mediated by a different spatial arrangement of collagen fibers. Such a mechanism, together with the estrogen mechanism of inhibition of osteoclasts mediated by cytokine (18), could explain the rapid marked bone loss observed in trabecular bone following estrogen depletion. Direct evidence of this hypothetical mechanism is not available at

\section{References}

1. Michalsky M, Noris Suarez K, Bettica P, Pecile A, Moro L. Rat cortical and trabecular bone collagen glycosylation are differently influenced by ovariectomy. Biochem Biophys Res Commun 1993; 192:1281-8.

2. Torre-Blanco A, Adachi E, Hojima Y, Wootton JAM, Minor RR, Prockop DJ. Temperature-induced post-translational overmodification of type 1 procollagen. J Biol Chem 1992; 267:2650-5.

3. Barengolts EI, Gajardo HF, Rosol TJ, D'Anza JJ, Pena M, Botsis $J$, et al. Effects of progesterone on postovariectomy bone loss in aged rats. J Bone Miner Res 1990; 5:1143-7.

4. Wink CS, Felts WJL. Effects of castration on bone structure of male rats: a model of osteoporosis. Calcif Tissue Int 1980; $32: 77-82$.

5. Kalu DN, Salerno E, Liu CC, Echon R, Ray M, Garza-Zapata $M$, et al. A comparative study of the actions of tamoxifen, estrogen and progesterone in the ovariectomized rat. Bone Miner 1991; 15:109-24.

6. Turner RT, Wakley GK, Hannon KS, Bell NH. Tamoxifen inhibits osteoclast-mediated resorption of trabecular bone in ovarian hormone-deficient rats. Endocrinology 1988; 122:1146-50. the moment. An indirect observation on the relationship among estrogens, bone collagen glycosylation and mechanical properties of bone, could come from osteogenesis imperfecta. One of the biochemical defects of this disease is over-glycosylation of the collagen molecule which leads to a decrease in fibril diameter (19). Interestingly, in a mild form of osteogenesis imperfecta, the rate of bone fractures correlates negatively with estrogen status; i. e. it decreases at puberty and increases during pregnancy and after menopause (19).

Moreover these results, backed up by those recently obtained in the rat (12), which showed that type I collagen of cortical and trabecular bone differs in the extent of glycosylation and cross-link formation (collagen of trabecular bone appears to be more glycosylated than collagen of cortical bone which, on the contrary, appears to be more cross-linked), may suggest a new interpretation in using bone markers. Were these data to be confirmed in humans, it might be possible to distinguish bone resorption in trabecular and cortical compartments.

\section{Conclusion}

We have shown once again that trabecular bone collagen glycosylation increases in rats following ovariectomy. Treatment of the ovariectomized animals during the experiment (45 days, i. e. from 100 to 145 days of age), either with $17 \beta$-estradiol or tamoxifen, prevented trabecular collagen over-glycosylation, indicating that estrogens affect this collagen post-translational modification.

\section{Acknowledgements}

The research was supported by the Italian Ministry of University and of Scientific and Technological Research and Research National Council.

7. Bailey AJ, Wotton SF, Sims TJ, Thompson PW. Biochemical changes in the collagen of human osteoporotic bone matrix. Conn Tissue Res 1993; 29:119-32.

8. Teerlink T, Tavenier $P$, Netelonbos $C$. Selective determination of hydroxyproline in urine by high-performance liquid chromatography using precolumn derivatization. Clin Chim Acta 1989; 183:309-16.

9. Moro L, Modricky C, Stagni N, Vittur F, de Bernard B. Highperformance liquid chromatographic analysis of urinary hydroxylysyl glycosides as indicators of collagen turnover. Analyst 1984; 109:1621-2.

10. Gray WR. Dansyl chloride procedure. Methods Enzymol 1967; 11:149-51.

11. de Bernard B, Moro L, Gazzarrini C, Battista C. Galactosylhydroxylysine as biochemical marker of bone collagen degradation. In: De Bastiani G, Pecile A, Pietrogrande V, Torri G, editorts. Le osteoporosi e il loro trattamento. Bologna: Monduzzi; 1989:9-15.

12. Noris Suarez K, Romanello M, Bettica P, Moro L. Collagen type I of rat cortical and trabecular bone differs in the extent of post-translational modifications. Calcif Tissue Int 1996; 58:76-9. 
13. Manolagas SC, Jilka RL. Cytokines, hematopoiesis, osteoclastogenesis, and estrogens. Calcif Tissue Int 1992; 50:199-202.

14. Bailey AJ, Wotton SF, Sims TJ, Thompson PW. Post-translational modifications in the collagen of human osteoporotic femoral head. Biochem Biophys Res Commun 1992; 185:801-5.

15. Bätge B, Diebold J, Stein H, Bodo M, Müller PK. Compositional analysis of the collagenous bone matrix. A study on adult normal and osteopenic bone tissue. Eur $\mathrm{J}$ Clin Invest 1992; 22:805-12.

16. Landis WJ. The strength of a calcified tissue depends in part on the molecular structure and organization of its constituent mineral crystals in their organic matrix. Bone 1995; 16:53344.

17. Yang C, Niu C, Bodo M, Gabriel E, Notbohm H, Wolf E, et al. Fulvic acid supplementation and selenium deficiency dis- turb the structural integrity of mouse skeletal tissue. An animal model to study the molecular defects of Kashin-Beck disease. Biochem J 1993; 289:829-35.

18. Manolagas SC, Jilka RL. Mechanisms of disease: bone marrow, cytokines, and bone remodeling - emerging insights into pathophysiology of osteoporosis. N Engl J Med 1995; 332:305-11.

19. Prockop DJ. Heritable disorders of connective tissue. In: Braunwald E, Isselbacher KJ, Petersdorf RG, Wilson JD, Martin JB, Fauci AS, editors. Principles of international medicine. New York: Hill Book Company, 11 th ed, vol 2; 1987:1683-5.

\section{Received August 1/October 9, 1996}

Corresponding author: Luigi Moro, $\mathrm{MD}, \mathrm{PhD}$, Dipartimento di Biochimica, Biofisica e Chimica delle Macromolecole, Università degli Studi di Trieste, Via L. Giorgieri 1, I-34127 Trieste, Italy 
